\title{
An Improved Structural Analysis Method for Isolator with Quasi-Zero-Stiffness Characteristic
}

\author{
Wei Zhang, Xiaoping Li ${ }^{D}$, Jian Li, and Xiqiu Li \\ State Key Laboratory of Digital Manufacturing Equipment and Technology, Huazhong University of Science and Technology, \\ Wuhan 430074, China \\ Correspondence should be addressed to Xiaoping Li; lixiaoping@hust.edu.cn
}

Received 25 March 2021; Revised 26 July 2021; Accepted 12 November 2021; Published 15 December 2021

Academic Editor: Jiaxi Zhou

Copyright (c) 2021 Wei Zhang et al. This is an open access article distributed under the Creative Commons Attribution License, which permits unrestricted use, distribution, and reproduction in any medium, provided the original work is properly cited.

A typical quasi-zero-stiffness (QZS) vibration isolator consisting of a vertical spring and two oblique springs has been widely researched on its static and dynamic characteristics. A general criterion for determining structural parameters of QZS isolator is to achieve low nondimensional stiffness around the equilibrium position. However, lower nondimensional stiffness of linear isolator means lower isolation frequency, which may be invalid on QZS isolator. Because there is an implicit relationship between geometric parameter and stiffness ratio of QZS isolator, this study presents an improved optimization criterion for determining the optimal structural parameters of the typical QZS isolator. The optimization criterion is that the QZS isolator has the maximum displacement range around the equilibrium position without exceeding given natural frequency, rather than given nondimensional stiffness. The results show that isolator with these optimal parameters can achieve lower stiffness around the equilibrium position and better vibration isolation performance. Furthermore, an extended QZS isolator consisting of vertical spring with fixed stiffness and prestressed oblique springs is discussed to further improve stiffness characteristic. Better stiffness performance can be obtained when the prestressed oblique springs have softening stiffness and the exponent of the nonlinear stiffness is 2. Considering the existence of friction in practical application, the influence of friction on both static and dynamic characteristics is investigated. The analysis reveals that friction has little influence on its stiffness characteristic around the static equilibrium position and friction damping produced by friction affects the response amplitude and resonant frequency in dynamics.

\section{Introduction}

Vibration isolator has been widely applied in manufacturing equipment such as semiconductor devices and optical instruments for its technical advantage. It is well known that vibration isolation occurs above frequency of $\sqrt{2} \omega_{n}$, where $\omega_{n}$ is natural frequency of isolator, and vibration isolation performance can be improved by reducing the natural frequency. However, a lower natural frequency results in a larger static deflection for a given load, namely a weakened load capacity. Therefore, there is a dichotomy between natural frequency and load capacity, which is an important issue of vibration isolator. This contradiction can be resolved well by a quasi-zero-stiffness (QZS) mechanism with high static stiffness and low dynamic stiffness [1].
In the last decade, the theoretical analysis, design, and experimental research on QZS isolator have been widely reported. The QZS mechanism mainly includes two parts: positive stiffness part and negative stiffness part. The most typical three-spring isolator, as shown in Figure 1, consists of a vertical spring and two inclined springs in parallel. The vertical spring provides large bearing capacity, and two inclined springs act as negative stiffness spring as a whole in the vertical direction to reduce the dynamic stiffness of the mechanism. One fundamental and important work is to determine the parameters of the mechanism, such as geometric parameter and stiffness of its elastic elements. Carrella et al. [2] have first proposed a quantitative static analysis method on the QZS system with three springs and provided the best match relationship between the angle and the stiffness 


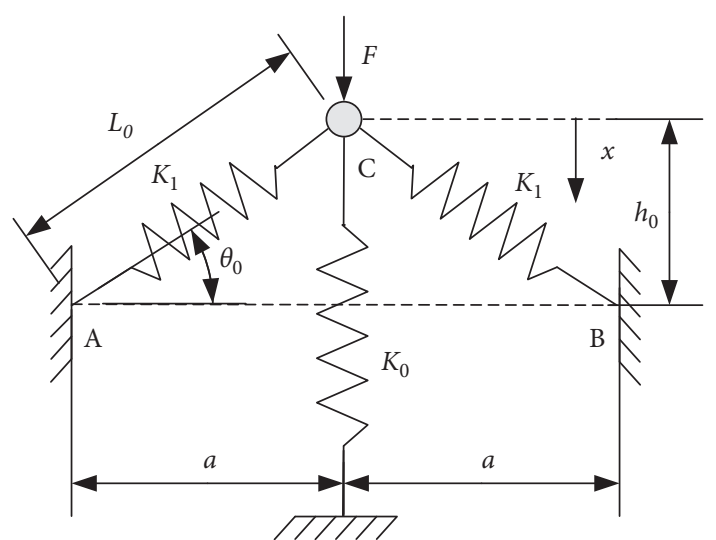

FIGURE 1: Schematic representation of a typical QZS mechanism with a vertical spring and two oblique springs.

ratio of vertical and oblique springs to achieve low stiffness around the static equilibrium position. Kovacic et al. [3] conducted follow-up research by taking two nonlinear prestressed oblique springs into consideration and obtained lower stiffness of the QZS system than that with linear stiffness oblique springs. Subsequently, a further dynamic analysis of this QZS mechanism with three springs was conducted [4]. Wang et al. [5] introduced stiffness range parameter and static equilibrium position stiffness to indicate different stiffnesses of the nonlinear isolator and investigated the influence of these parameters on dynamic responses and vibration isolation performance. Liu et al. [6] analysed the performance of the nonlinear isolator excited by different displacement shock excitations from the base and found that the performance was related to the parameters of the nonlinear isolator and the type of the shock excitation. It is beneficial to introduce a QZS vibration isolator for shock excitation, and the performance is limited by certain conditions. Wang et al. [7] employed the probabilistic linearization method to determine the dynamic response of the QZS vibration isolator under random excitation and evaluated the isolation performance with the equivalent linear vibration isolator. In addition to research of three-spring isolator under different types of external disturbance, relevant literature involves the exploration of various three-spring extension systems to further improve its performance. A bioinspired nonlinear system with four linear springs arranged in a K-shaped configuration was proposed for the purpose of potential energy maximization [8]. Zhao et al. [9-11] proposed new limb-like QZS systems with two pairs and three pairs of oblique springs to enlarge the QZS range and isolation frequency band. Similarly, Yan et al. [12] proposed a large stroke QZS vibration isolator using a three-link mechanism, which is less sensitive to vibration amplitude than the traditional QZS isolator. Thanh and Vu [13] devised an adjustable vibration isolator to increase the load-bearing capacity and maintain the isolation effectiveness when the isolated weight is changed. In addition to a single-stage isolator, a two-stage isolator with oblique springs has also been studied. Lu et al. [14-16] studied a two-stage vibration isolation system with nonlinear stiffness and investigated the effects of nonlinear stiffness and damping of two stages on vibration transmissibility. Wang et al. [17] compared the dynamic performance of single- and two-stage QZS isolators, and the results showed that the two-stage vibration isolator can be tuned to achieve improved isolation performance in the higher frequency region than the baseline vibration isolators. The ideal QZS isolator has the lowest stiffness at the equilibrium position where the oblique spring is horizontal. When mass load is changed, static equilibrium position and dynamic stiffness of isolator are changed accordingly. Abolfathi et al. [18] analysed the dynamic characteristics of isolator with load error. The analysis showed that although the mistuned case degraded the performance of the isolator compared with the perfectly tuned case, it still performed better than the corresponding linear isolator provided that the amplitude of excitation is not too large. Moreover, Shaw et al. [19] found that the performance of a mount with a symmetric stiffness-displacement relationship is highly sensitive to load error and a mount with an asymmetric stiffness-displacement relationship can offer significant performance advantage in this situation. Besides, typical oblique coiled springs were used to provide negative stiffness. Some other forms of negative stiffness structure are proposed and studied, such as disk spring [20, 21], notched flexure [22], magnetic spring [23-25], Euler buckled beam [26-28], cam roller spring [29-31], scissor-like structure [32-34], and other novel structures [35-37]. Different negative stiffness elements have their advantages. Disk spring and Euler buckled beam can offer great support capacity with small deflection. Cam roller spring and scissor-like structure can provide designed nonlinear stiffness through reasonable structural parameters. Negative stiffness provided by magnetic spring does not need direct contact, and the design of isolator can be flexible. The damping of QZS isolator affects its resonant frequency and dynamic characteristic. Some related work has been carried out to analyse and improve the vibration isolation performance. Hao et al. [38] proposed QZS isolator with bilateral damping in parallel with vertical spring. By adapting bilateral damping constraint strategy, better vibration isolation behaviour can be achieved at both low and high frequencies. Lu et al. [15] studied the single-stage and two-stage isolators with nonlinear damping, which is achieved by configuring linear dampers and shows better performance at high frequency than those with linear viscous damping. In addition to damping produced by damper, the friction of QZS isolator during vibration produces damping and causes energy dissipation, which should be considered in practical application. Sun et al. [39, 40] considered the structural rotational friction of the joints and horizontal friction during sliding on the track in their analysis of the scissor-like structured platform. Ahn [41] put forward a movable magnet track for a linear motor stage, and friction and cable stiffness of which are taken into account during the mover motion. Besides, Li et al. [42] proposed a novel type of constant force compression mechanism and considered the friction between cam and spring sliders. The results show that the prototype has satisfactory characteristics as with the design requirement under both micro-friction and nonignorable conditions. 
Of all these QZS isolators mentioned above, the threespring QZS isolator is most widely studied as a classical structural model. The design and analysis methods applied to the three-spring isolator provide strong guide to various extended QZS isolators and isolators with different negative stiffness elements. A good QZS isolator has low dynamic stiffness around the equilibrium position and large load-bearing capacity. So, a general criterion for determining structural parameters of QZS isolator in previous papers is to achieve low nondimensional stiffness around the equilibrium position, but there is a difference between QZS isolator and linear isolator. Lower nondimensional stiffness of linear isolator means lower isolation frequency, which may not be true on QZS isolator, because there is an implicit relationship between geometric parameter and stiffness ratio of QZS isolator. So, an improved optimization criterion for determining the optimal structural parameters of the typical QZS isolator is proposed and the optimization criterion is to achieve the maximum displacement range without exceeding the given frequency. The nonlinear and prestressed oblique springs of the QZS system were studied by introducing two extended variables of structural parameters in previous work, but the effects of every extended parameter on static performance remain unclear when these parameters take different values. According to the previous work and in a more general case, an extended QZS isolator consisting of vertical spring with fixed stiffness and prestressed oblique springs is discussed to further improve stiffness characteristic. In addition, when the QZS isolator vibrates, there is friction produced by rotating joint in practical application and it may affect the vibration isolation performance. The influence of friction on both static and dynamic characteristics is investigated in this study. This research can provide some guidelines in the design of the typical QZS isolator and other complex QZS isolators.

The study is organized as follows: first, an improved optimization method for structural parameters of the typical QZS isolator is proposed; second, the static characteristics of the parameters of the extended QZS isolator are analysed; then, the influence of the friction on the stiffness and dynamic characteristics of QZS mechanism is discussed; and finally, the conclusions are presented.

\section{Optimization Criteria for Typical QZS Mechanism}

2.1. Stiffness of Typical QZS Mechanism. As shown in Figure 1, a typical isolator with high-static-low-dynamic-stiffness (HSLDS) consists of a vertical spring and two oblique springs, whose stiffness coefficients are $K_{0}$ and $K_{1}$, respectively. The positions of two oblique springs are symmetric compared with that of a vertical spring, and the distance between their two hinged ends is $2 a$. The initial support point $C$ is at the height of $h_{0}$ from $A$ and $B$, and two oblique springs have an initial length of $L_{0}$ and an angle of $\theta_{0}$ with respect to the horizontal direction. If the support force of the mechanism $F$ is nondimensionalized by $K_{0} a$, then the nondimensional force $\widehat{F}$, varying with the vertical nondimensional displacement of the support point $\hat{x}$, can be given by the following equation:

$$
\widehat{F}=\widehat{x}+2 \alpha(\gamma-\widehat{x})\left[\frac{\sqrt{1+\gamma^{2}}}{\sqrt{\hat{x}^{2}-2 \gamma \widehat{x}+1+\gamma^{2}}}-1\right],
$$

where $\hat{x}=x / a, \alpha=K_{1} / K_{0}$, and $\gamma=h_{0} / a$. The nondimensional stiffness is obtained by differentiating equation (1) with respect to $\hat{x}$.

$$
\widehat{K}=1+2 \alpha\left[1-\frac{\sqrt{1+\gamma^{2}}}{\left(\hat{x}^{2}-2 \gamma \hat{x}+1+\gamma^{2}\right)^{3 / 2}}\right] .
$$

According to the symmetry of the mechanism, the minimum value of $\widehat{K}$ is at the equilibrium position where the displacement $\widehat{x}_{e}$ satisfies the following equation:

$$
\widehat{x}_{e}=\gamma \text {. }
$$

Combining equation (2) with equation (3) and taking into account the QZS condition, the relationship between structural parameters $\alpha$ and $\gamma$ of QZS mechanism is given as follows:

$$
\alpha=\frac{1}{2\left(\sqrt{1+\gamma^{2}}-1\right)} \text {. }
$$

So equation (2) becomes as follows:

$$
\widehat{K}_{\mathrm{qzs}}=1+\frac{1}{\sqrt{1+\gamma^{2}}-1}\left[1-\frac{\sqrt{1+\gamma^{2}}}{\left(\hat{x}^{2}-2 \gamma \hat{x}+1+\gamma^{2}\right)^{3 / 2}}\right] \text {. }
$$

There is only one independent geometric parameter $\gamma$ to be determined in equation (5), and the stiffness ratio parameter $\alpha$ can be calculated by equation (4). When oblique spring is horizontal, it has maximum compression length. The deformation ratio of oblique spring at equilibrium is $\eta$, which satisfies the following equation:

$$
\eta=1-\frac{1}{\sqrt{1+\gamma^{2}}}
$$

The value of $\eta$ increases monotonously with the increase in $\gamma$, and the maximum value $\eta_{m}$ is the limit deformation ratio to behave linear stiffness. So, the geometric parameter $\gamma$ ranges between $\left(0, \sqrt{1 /\left(1-\eta_{m}\right)^{2}-1}\right)$, and the optimal values of $h_{0}$ and $L_{0}$ are then found in the interval (0, $a$ $\left.\sqrt{1 /\left(1-\eta_{m}\right)^{2}-1}\right)$ and $\left(0, a /\left(1-\eta_{m}\right)\right)$, respectively. The value of $\eta_{m}$ is determined by the structure of the coil spring, such as pitch and diameter. If $\eta_{m}$ is 0.5 , then the range of $\gamma$ is $(0, \sqrt{3})$.

2.2. Optimization Criteria for QZS Mechanism. The optimal structural parameters can be obtained by the trade-off between vibration isolation performance and load capacity. The optimization of QZS mechanism is to make it have the lowest natural frequency near the equilibrium point, which 
can be equivalent to the maximum displacement from the equilibrium point at a given frequency. In addition, the stiffness of vertical spring $K_{0}$ is a decisive parameter to determine its load capacity and a relatively higher value of $K_{0}$ is beneficial.

In an ideal condition, the QZS mechanism subjected to mass load stays at the equilibrium position where the oblique springs move along their axial direction, and the support force entirely comes from the vertical spring. The support force $F_{0}$ at the equilibrium position is given by the following equation:

$$
F_{0}=K_{0} a \gamma=M g,
$$

where $M$ is the rated load. The natural frequency of the QZS mechanism can be expressed as follows:

$$
f=\frac{1}{2 \pi} \sqrt{\frac{K}{M}}
$$

where $K$ is the stiffness of the QZS mechanism and yields the following:

$$
K=\widehat{K} K_{0}
$$

By substituting equations (7) and (9) into equation (8), the natural frequency of the QZS mechanism can be rewritten as follows:

$$
f=\frac{1}{2 \pi} \sqrt{\frac{\widehat{K} g}{a \gamma}}
$$

Equation (10) indicates that the geometric parameter $\gamma$ is contained in the expression of the natural frequency with respect to the nondimensional stiffness. To achieve expected performance, the natural frequency of the isolator should be constrained during operation. The optimization criterion is the realization of the maximum displacement from the equilibrium position at a given natural frequency.

If the QZS mechanism has a critical frequency $f_{c}$, then the critical nondimensional displacement of the support point $\hat{x}_{c}$ can be expressed as follows:

$$
\widehat{x}_{c}=\hat{x}_{e} \pm \hat{x}_{d}
$$

where $\widehat{x}_{d}$ is the excursion from the equilibrium position. The nondimensional movement range of the isolator $\widehat{d}_{f}$ at the critical natural frequency $f_{c}$ is twice the value of $\hat{x}_{d}$. By substituting equations (10) and (11) into equation (5), its expression can be written as follows:

$$
\hat{d}_{f}=2 \widehat{x}_{d}=2 \sqrt{\left[\frac{g \sqrt{\gamma^{2}+1}}{\left(g-4 \pi^{2} f_{c}^{2} a \gamma\right) \sqrt{\gamma^{2}+1+4 \pi^{2} f_{c}^{2} a \gamma}}\right]^{2 / 3}-1 .}
$$

The physical quantities $a$ and $f_{c}$ in equation (12) are determined by the demands of the isolator and regarded as constants here. The curves of nondimensional movement range with respect to the geometric parameter are plotted in Figure 2, where the nondimensional movement range $\widehat{d}_{f}$ increases monotonically with the increase in the geometric

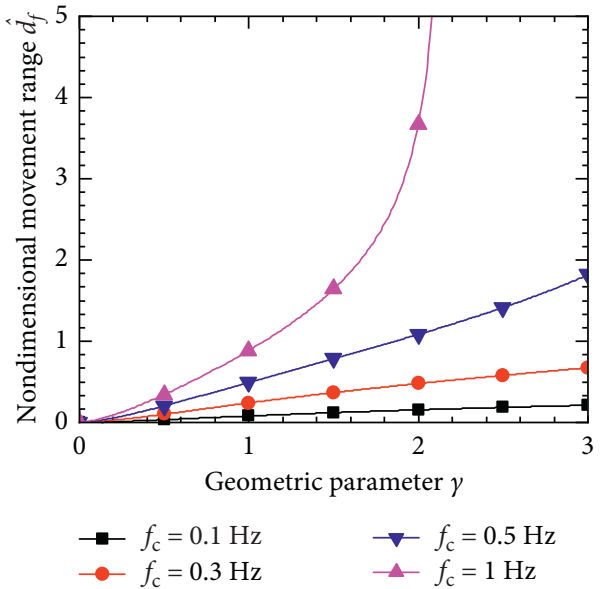

Figure 2: Nondimensional movement range versus the geometric parameter at different given critical frequencies when $a=0.2 \mathrm{~m}$.

parameter $\gamma$ at the same critical natural frequency. In the range of $\left(0, \sqrt{1 /\left(1-\eta_{m}\right)^{2}-1}\right)$, a higher value of $\gamma$ means a lower natural frequency of the mechanism near the equilibrium position. When the critical natural frequency $f_{c}$ is close to $0 \mathrm{~Hz}$, the nondimensional movement range $\widehat{d}_{f}$ is small and increases approximately linearly with the increase in the geometric parameter $\gamma$. When $f_{c}$ appropriate $1 \mathrm{~Hz}, \widehat{d}_{f}$ changes rapidly for its strong nonlinearity.

The optimal geometric parameters cannot be obtained by the QZS condition, for it has no upper limit. It is necessary to propose another constraint considering load capacity. The QZS isolator usually uses a regulating unit to adjust the support point to the initial equilibrium position $D$ when bearing different loads, as shown in Figure 3.

Supposing that the adjustable height is $2 \Delta h$ and the load is from $M-\Delta M$ to $M+\Delta M$, then these parameters satisfy the following equation:

$$
K_{0} \Delta h=\Delta M g .
$$

Combining equations (7) and (13), $\Delta M$ can be expressed as follows:

$$
\Delta M=\frac{M \Delta h}{a \gamma}
$$

Equation (14) reveals that a lower value of $\gamma$ results in a higher value of $\Delta M$ for a given $a, \Delta h$, and $M$, which can provide a larger load capacity. Thus, a lower value of the geometric parameter $\gamma$ should be preferred in view of load capacity. Combined with the characteristics of vibration isolation and load bearing, the optimum value of the geometric parameter of the QZS mechanism can be determined by equations (12) and (14). If only isolation performance is considered, a better passive isolation performance can be achieved by increasing the value of the geometric parameter at the expense of load capacity and vice versa.

When the dimension of the QZS mechanism is limited and $L_{0}$ is a constant, the curves of the movement range $d_{f}$ with respect to the geometric parameter $\gamma$ are plotted in 


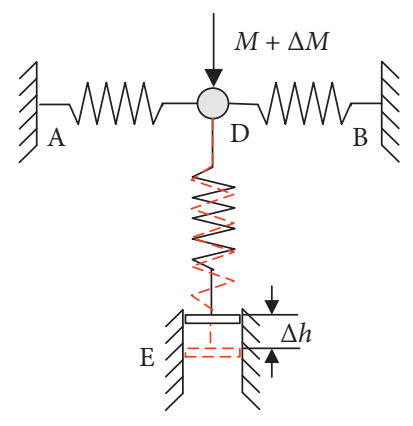

(a)

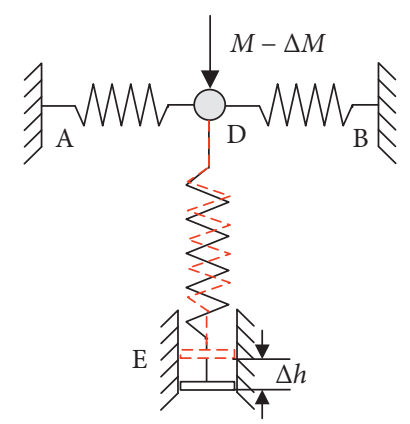

(b)

Figure 3: Adjustable height of the regulating unit $\Delta h$ from the initial position $E$ with load range $\Delta M$.

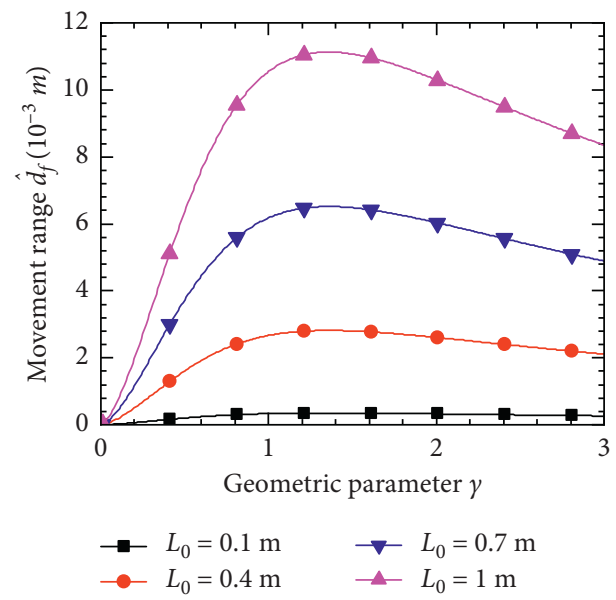

FIgURE 4: Movement range versus geometric parameter when the critical frequency is $0.01 \mathrm{~Hz}$.

Figure 4. It reveals that $d_{f}$ has a maximum value when $\gamma_{\mathrm{opt}}$ is 1.36 and $L_{0}$ is in the range $(0,1]$. According to the QZS condition given in equation (4), the corresponding parameter $\alpha_{\text {opt }}$ is 0.73 .

Based on analysis by Carrella et al. [2], the optimum structural parameters denoted by the letters with apostrophe are $a^{\prime} / L_{0}^{\prime}=2 / 3$ (namely, $\gamma_{\text {opt }}{ }^{\prime}=1.12$, nondimensional by $a$ ) and $\alpha_{\text {opt }}{ }^{\prime}=1$. Without loss of generality, let $L_{0}=L_{0}^{\prime}=0.4 \mathrm{~m}$. The curves of stiffness and natural frequency with respect to displacement from the equilibrium position under the two sets of optimum structural parameters are plotted in Figure 5. It demonstrates that both stiffness and natural frequency of the QZS system, designed by the optimization method presented in this study, are lower than those in the previous method under the same displacement. Moreover, their difference increases when the support point moves away from the equilibrium position.

If the QZS system is subjected to a small harmonic disturbance, its response is very small and is approximate to a linear system. The curves of vibration transmissibility at two different natural frequencies are plotted in Figure 6. It is obvious that the system with a smaller resonant frequency has better vibration isolation performance. If the harmonic disturbance is large, the QZS system has nonlinear behaviour. The equation of motion can be approximated by Duffing's equation with linear and nonlinear terms $b_{1}$ and $b_{2}$ and nondimensionalized by $K_{0} a$ as follows:

$$
\frac{1}{\omega_{0}^{2}} \widehat{x}^{\prime \prime}+\frac{2 \xi}{\omega_{0}} \widehat{x}^{\prime}+b_{1} \widehat{x}+b_{2} \widehat{x}^{3}=\widehat{F} \cos (\omega t+\phi),
$$

where $\omega_{0}=\sqrt{K_{0} / M}, \xi=c / 2 \sqrt{K_{0} M}, \quad b_{1}=1-2 \alpha\left(\sqrt{1+\gamma^{2}}\right.$ -1 ), and $b_{2}=\alpha \sqrt{1+\gamma^{2}}$, and $\xi$, $\omega$, and $\widehat{F}$ are damping ratio, disturbance angular frequency, and nondimensional disturbance force, respectively. An approximate solution is obtained by applying the harmonic balance method. The disturbance force $\widehat{F}$ and the response magnitude $A$ have the relationship as follows:

$$
\widehat{F}^{2}=\left[\left(b_{1}-\Omega^{2}\right) A+\frac{3}{4} b_{2} A^{3}\right]^{2}+[2 \xi \Omega A]^{2},
$$

where $\Omega=\omega / \omega_{0}$. When isolator satisfies QZS condition, the value of parameter $b_{1}$ is 0 and equation (16) reduces to the following:

$$
\widehat{F}^{2}=\left(-\Omega^{2} A+\frac{3}{4} b_{2} A^{3}\right)^{2}+(2 \xi \Omega A)^{2} .
$$

Equation (17) reveals that response amplitude $A$ is related to disturbance force $\widehat{F}$, angular frequency ratio $\Omega$, and nonlinear term $b_{2}$. The response magnitude curves with different optimal $\gamma$ are plotted in Figure 7. It demonstrates that the resonant peak slopes to the right side due to the nonlinear term $b_{2}$, and the peak frequency with $\gamma=1.36$ is lower than the one with $\gamma=1.13$. According to equation (7), the angular frequency ratio can be expressed as follows:

$$
\Omega=\sqrt{\frac{3}{4} b_{2} A^{2}-2 \xi^{2} \pm \frac{1}{A} \sqrt{4 \xi^{4} A^{2}-3 b_{2} \xi^{2} A^{4}+\widehat{F}^{2}}}
$$

The angular frequency at the peak value has unique solution, which satisfies the following equation:

$$
4 \xi^{4} A^{2}-3 b_{2} \xi^{2} A^{4}+\widehat{F}^{2}=0 .
$$

The resulting angular frequency ratio of the quasi-zero stiffness isolator is determined by the following equation:

$$
\Omega_{r}=\sqrt{\frac{3}{4} b_{2} A_{r}^{2}-2 \xi^{2}},
$$




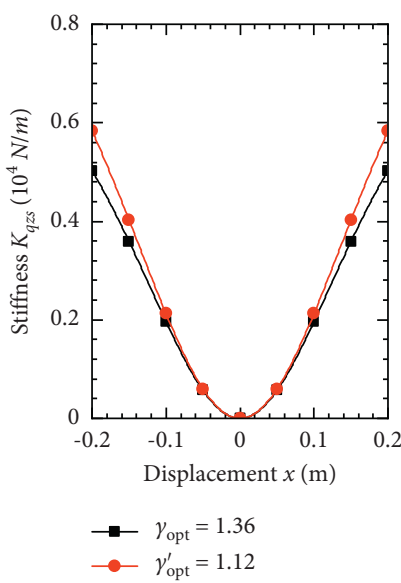

(a)

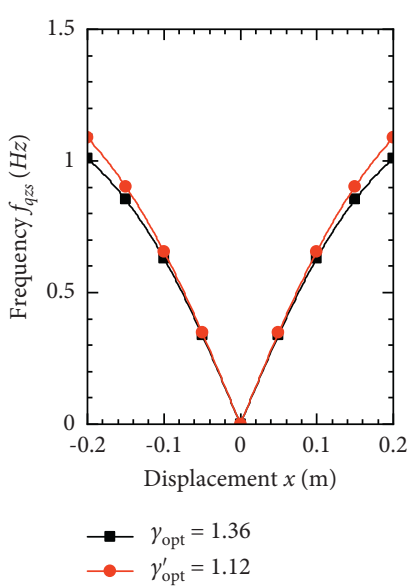

(b)

FIGURE 5: (a) Stiffness versus displacement from the equilibrium position. (b) Natural frequency versus displacement from the equilibrium position.

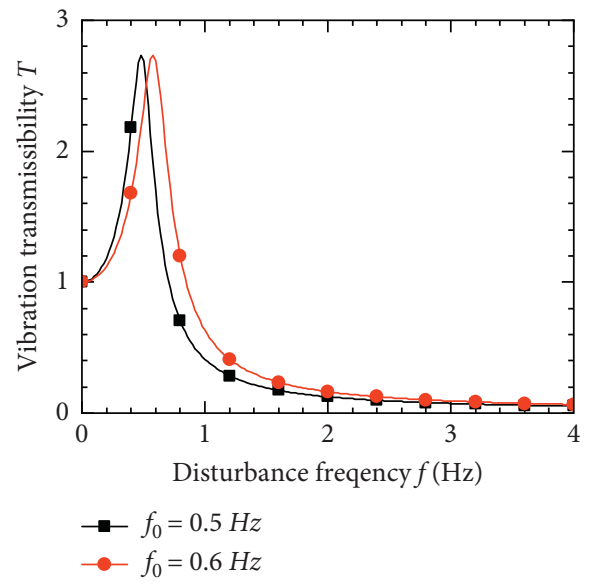

FIGURE 6: Vibration transmissibility versus disturbance frequency at damping ratio 0.2 .

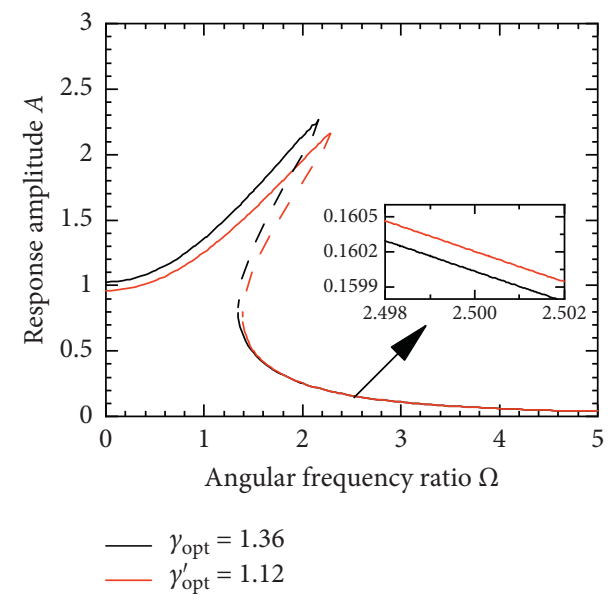

FIGURE 7: Response amplitude versus angular frequency ratio when $\widehat{F}=1$ and $\xi=0.1$. where $A_{r}$ is the peak response amplitude and determined by equation (19). The relationship between $\Omega$ and $\gamma$ is drawn in Figure 8 . The curve indicates that the resonant frequency of nonlinear mechanism becomes small with the increase in $\gamma$. So, a larger $\gamma$ is also beneficial to vibration isolation bandwidth in nonlinear condition, and the result is similar to linear mechanism.

As shown in Figure 5(a), smaller curvature corresponds to lower nonlinear term parameter $b_{2}$. The response magnitude curves with different $b_{2}$ are plotted in Figure 7, which demonstrates that the resonant peak slopes to the right side due to the nonlinear term. Despite the complex phenomenon around the resonant frequency, a lower vibration magnitude can be achieved with a weaker nonlinearity above the resonant frequency because its natural frequency is rather small. Then, a conclusion can be drawn that the QZS mechanism based on the optimization method in this study has better performance of vibration isolation despite small or large disturbance.

\section{Optimization Criteria for Extended QZS Mechanism}

There is one independent variable in the typical QZS isolator discussed above. As many negative stiffness structures are proposed, different nonlinear stiffness expressions are derived. Similarly, it deserves discussion on how to further improve the vibration performance through an extended three-spring isolator. The nonlinear and prestressed oblique springs of the QZS system were studied by introducing two extended variables of structural parameters [3]. It revealed that cubic softening nonlinear stiffness and prestressed oblique springs could further improve the vibration isolation behaviour with the optimum extended parameters, but the effects of every extended parameter on static performance remain unclear when these parameters take different values. According to the previous work and in a more general case, 


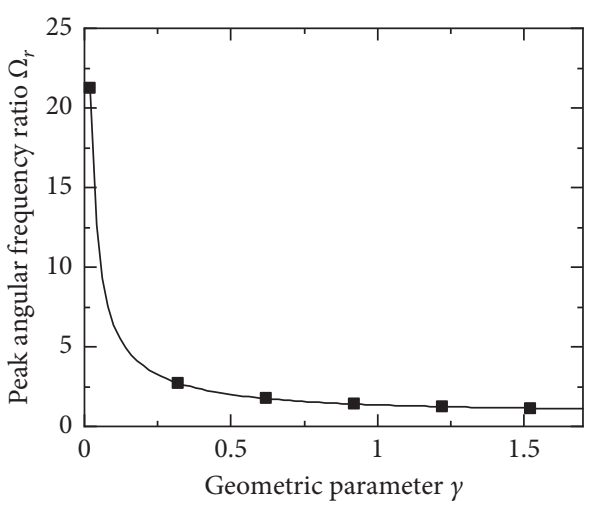

FIGURE 8: Peak angular frequency ratio versus geometric parameter when $\xi=0.1$ and $\widehat{F}=1$.

some extended forms of the simple QZS isolator, such as vertical spring with fixed stiffness and prestressed oblique springs with variable stiffness, are analysed and the effects of every structural parameter on the static characteristic are explored, respectively.

An extended form of the QZS isolator has an initial position where the vertical spring is in free length, as shown in Figure 9. Stiffness of the oblique spring is as follows:

$$
K=K_{1}-K_{2} x^{n-1} \text {, }
$$

where $K_{1}$ and $K_{2}$ are the coefficients of linear and nonlinear stiffness and $n$ is the order of nonlinear term. When the value of $K_{2}$ is positive or negative, the oblique springs have softening or hardening stiffness, respectively. The restoring force of oblique springs $F_{1}$ with respect to displacement $x$ is as follows:

$$
\begin{aligned}
F_{1}= & K_{1}\left(L_{0}-\sqrt{\left(h_{0}-x\right)^{2}+a^{2}}+\delta\right) \\
& -K_{2}\left(L_{0}-\sqrt{\left(h_{0}-x\right)^{2}+a^{2}}+\delta\right)^{n},
\end{aligned}
$$

where $\delta$ denotes the pre-compression length. Thus, the support force $F_{\text {ext }}$ of the extended QZS isolator can be written as follows:

$$
F_{\text {ext }}=K_{0} x+2 F_{1} \sin \theta,
$$

where $\sin \theta=\left(h_{0}-x\right) / L_{0}$. Two additional structural parameters are introduced as follows:

$$
\begin{aligned}
& \widehat{\delta}=\frac{\delta}{a}, \\
& \beta=\frac{K_{2} a^{n-1}}{K_{0}} .
\end{aligned}
$$

The dimensionless support force $\widehat{F}_{\text {ext }}$ and dimensionless stiffness $\widehat{K}_{\text {ext }}$ are nondimensionalized by $K_{0} a$ and $K_{0}$, respectively, and can be separately written as follows:

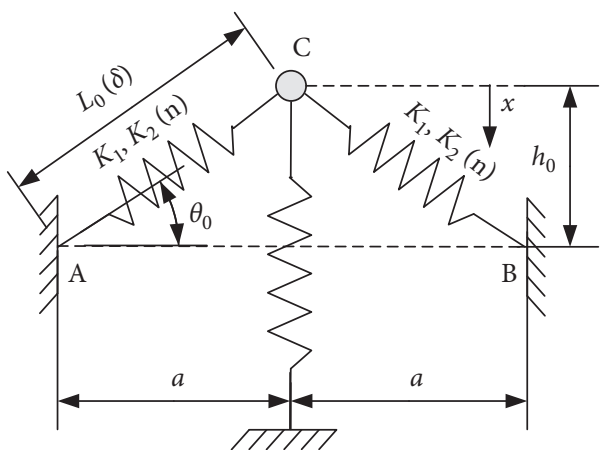

FIgURE 9: An extended QZS mechanism consisting of prestressed oblique springs with variable stiffness and vertical spring with fixed stiffness.

$$
\begin{aligned}
\widehat{F}_{\text {ext }}= & \widehat{x}+2 \alpha(\gamma-\widehat{x})\left(\frac{\sqrt{1+\gamma^{2}}+\widehat{\delta}}{\Lambda}-1\right) \\
& -2 \beta \frac{(\gamma-\widehat{x})}{\Lambda}\left(\sqrt{1+\gamma^{2}}+\widehat{\delta}-\Lambda\right)^{n},
\end{aligned}
$$

and

$$
\widehat{K}_{\text {ext }}=1+2 \alpha\left(1-\frac{F}{A^{3}}\right)-2 \beta \frac{\Delta^{\nu-1}}{A^{3}}\left[n A(\widehat{x}-\gamma)^{2}-\Delta\right],
$$

where $\Lambda=\sqrt{\widehat{x}^{2}-2 \gamma \widehat{x}+\gamma^{2}+1}, \Gamma=\sqrt{1+\gamma^{2}}+\widehat{\delta}, \Delta=\Gamma-\Lambda$.

It is difficult to derive the displacement range of the extended mechanism with all structural parameters at the critical natural frequency, so the influence of every parameter on the vibration isolation characteristic under the QZS condition is then analysed in statics.

3.1. Effect of Prestressed Oblique Springs. When the precompressed length of oblique springs is $\delta$ at initial position, the nondimensional movement range of the QZS mechanism $\widehat{d}_{1}$ at the critical nondimensional stiffness $\widehat{K}_{c}$ is given by the following equation:

$$
\widehat{d}_{1}=2 \sqrt{\left[\frac{\Gamma}{\left(1-\widehat{K}_{c}\right) \Gamma+\widehat{K}_{c}}\right]^{2 / 3}-1 .}
$$

The effect of the structural parameter $\widehat{\delta}$ on stiffness characteristic can be approximately equivalent to that of the structural parameter $\gamma$. It can be intuitively seen from Figure 10(a) that the initial support point $B$ of QZS mechanism with pre-compressed oblique springs can move to a new point $A$ by the resilience of the oblique springs. As the stiffness of vertical spring is constant, these two QZS mechanisms have the same stiffness characteristic. Suppose 


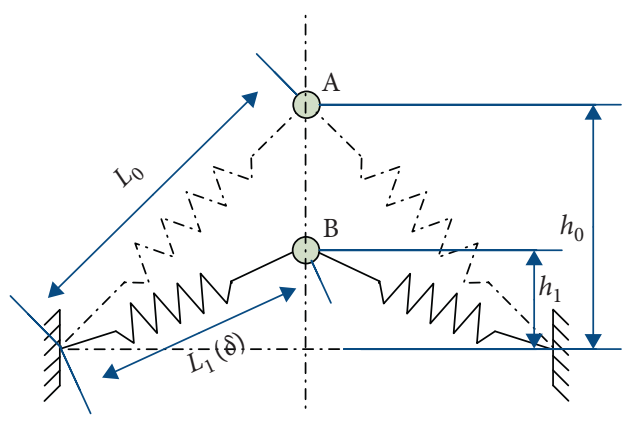

(a)

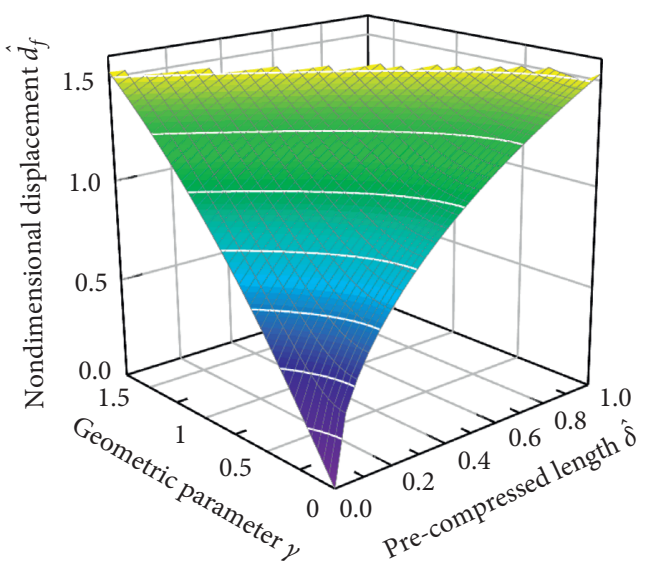

(b)

Figure 10: (a) Schematic representation of mechanism with prestressed oblique springs (point B) and free state oblique springs (point A). (b) Relationship among displacement of the QZS mechanism, geometric parameter, and nondimensional compression length at $f=0.5 \mathrm{~Hz}$ and $a=0.2 \mathrm{~m}$.

the geometric parameters of QZS mechanisms with initial positions $A$ and $B$ are $\gamma_{1}$ and $\gamma_{2}$, then $\widehat{\delta}$ satisfies as follows:

$$
\widehat{\delta}=\sqrt{1+\gamma_{1}^{2}}-\sqrt{1+\gamma_{2}^{2}}
$$

If the limit deformation ratio $\eta_{m}$ is 0.5 , the values of $\widehat{\delta}$ are not greater than 1 . The relationship between the nondimensional displacement $\widehat{d}_{1}$ and the structural parameters $\gamma$ and $\widehat{\delta}$ is illustrated in Figure 10(b). It shows that the increase in $\widehat{\delta}$, resulting in the increase in $\widehat{d}_{1}$, improves the frequency characteristic around the equilibrium position. Besides, considering the linear deformation range of oblique spring, a higher value of $\gamma$ reduces the available range of $\delta$. When $\gamma$ is
0 , the maximum $\widehat{\delta}$ can be obtained and the corresponding $\widehat{d}_{f}$ has the same value as the case that $\gamma$ has the maximum value. Therefore, when the oblique springs are prestressed, the QZS mechanism has a lower natural frequency around the equilibrium position. Moreover, a greater value of compression length brings about better isolation performance and a more compact dimension of the mechanism.

3.2. Effect of Oblique Springs with Variable Stiffness. When the oblique springs have nonlinear stiffness, the nondimensional stiffness of the QZS mechanism $\widehat{K}_{\text {qzs_- }}$ then can be given by the following equation:

$$
\widehat{K}_{\mathrm{qzs} \_1}=1+\frac{\Lambda^{3}-\sqrt{1+\gamma^{2}}}{\left(\sqrt{1+\gamma^{2}}-1\right) \Lambda^{3}}\left[1+2 \beta\left(\sqrt{1+\gamma^{2}}-1\right)^{n}\right]-\frac{2 \beta \Psi^{n-1}}{\Lambda^{3}}\left[n \Lambda(\widehat{x}-\gamma)^{2}-\Psi\right],
$$

where $\Psi=\sqrt{\gamma^{2}+1}-\Lambda$.

The expression of $K_{\mathrm{qzs} \_1}$ is complicated, and the effect of the extended parameters can be analysed qualitatively. The curves of the nondimensional stiffness and displacement for different $\beta$ and $n$ are illustrated in Figure 11. The nondimensional stiffness-displacement curves, shown in Figures 11(a)-11(c), gradually flatten near the equilibrium position and become steep at displacement away from the equilibrium position with the increase in $\beta$. Furthermore, as the exponent $n$ increases, the differences between the curves for different $\beta$ become smaller. A flatter nondimensional stiffness-displacement curve means a larger movement range of the mechanism at a given $\widehat{K}_{c}$, and the vibration isolation performance can be improved near the equilibrium position by introducing a stiffness parameter $\beta$ with positive value, particularly when $n$ is small. Likewise, Figures $11(\mathrm{~d})-$ 11(f) reveal that the nondimensional stiffness has the minimum value for the same $\beta$ near the equilibrium position when the value of $n$ is 2 . Besides, a larger $\beta$ is beneficial to increase the difference in values of $\widehat{K}_{\mathrm{qzs} \_1}$ for different $n$, which results in better vibration isolation performance. When $n$ is 2, the stiffness of oblique spring becomes the following:

$$
K=K_{1}-K_{2} x=K_{0}(\alpha-\beta \widehat{x}) .
$$

According to equation (30), the stiffness of oblique spring changes linearly with the displacement and the value of $\beta$ is constrained by the maximum displacement. In fact, the beneficial values of $\beta$ and $n$ are aimed to increase the rate of change in stiffness. If a larger $n$ is chosen, a relative larger $\beta$ can be considered to compensate for the weakness. Equation (24) indicates that the effect of $\beta$ and $n$ can be equivalent to each other, and this is the same with the case of $\widehat{\delta}$ and $\gamma$. Hence, the static analysis indicates that the vibration 


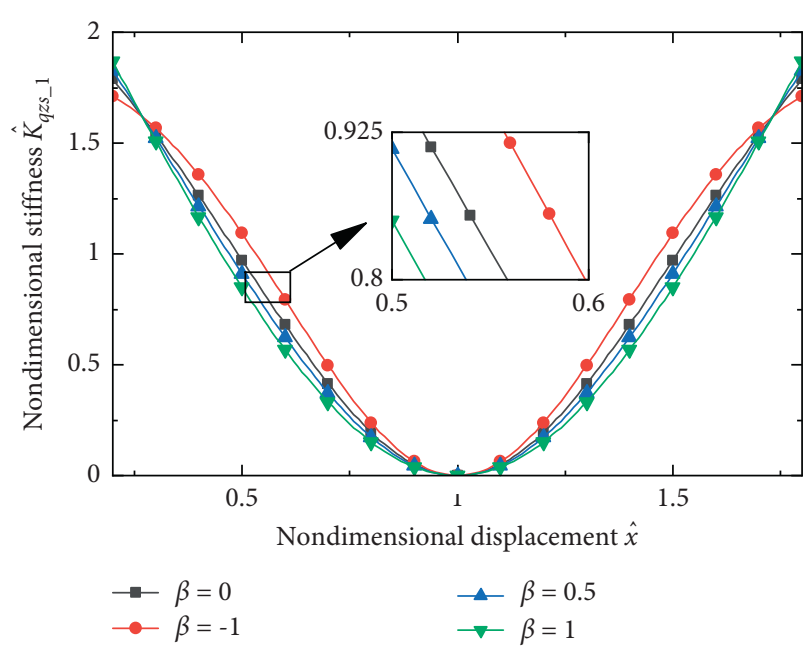

(a)

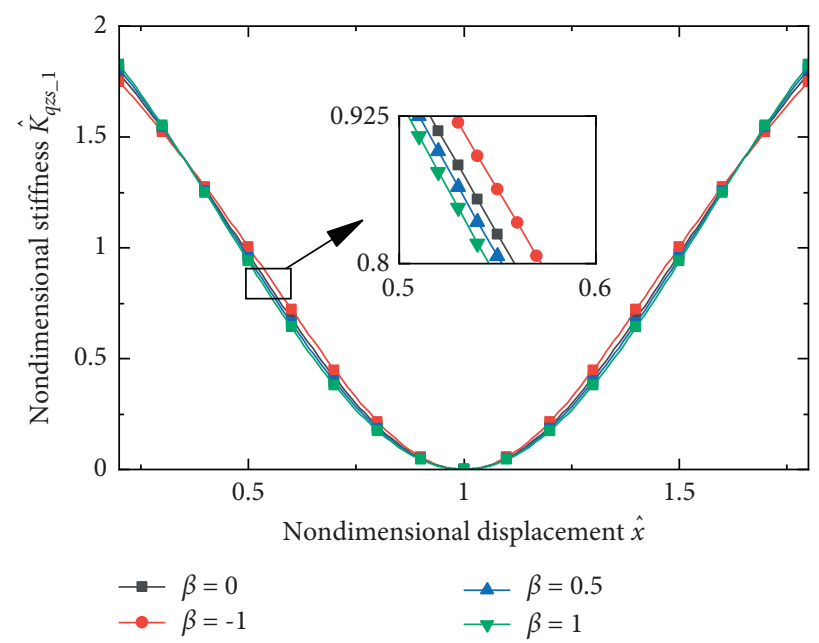

(c)

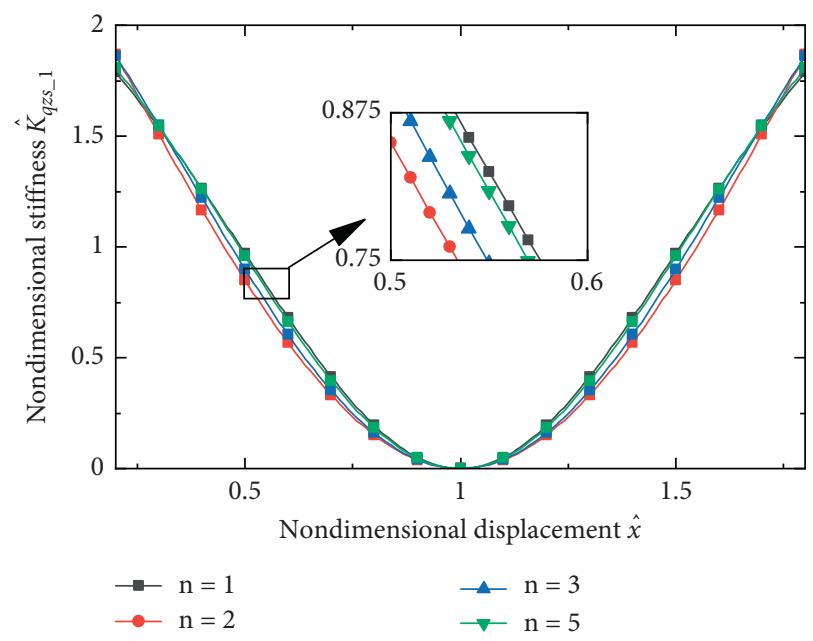

(e)

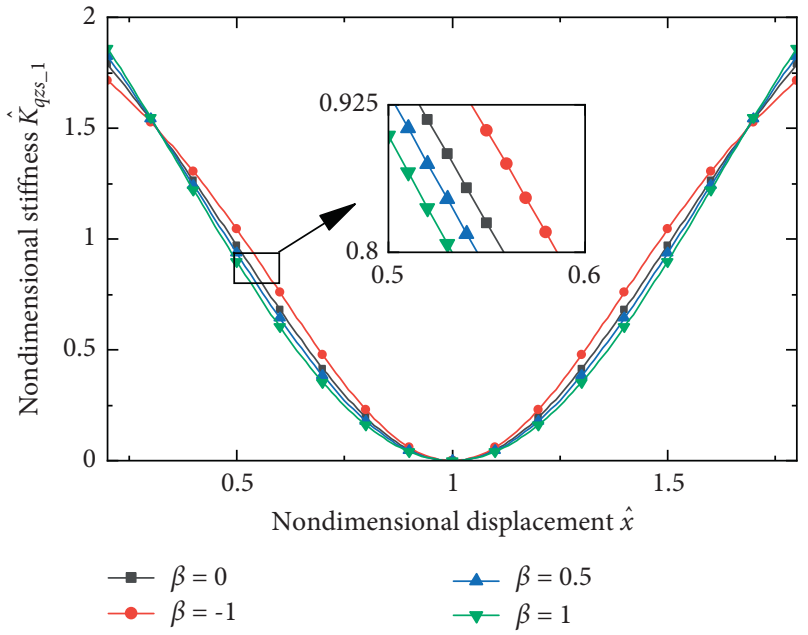

(b)

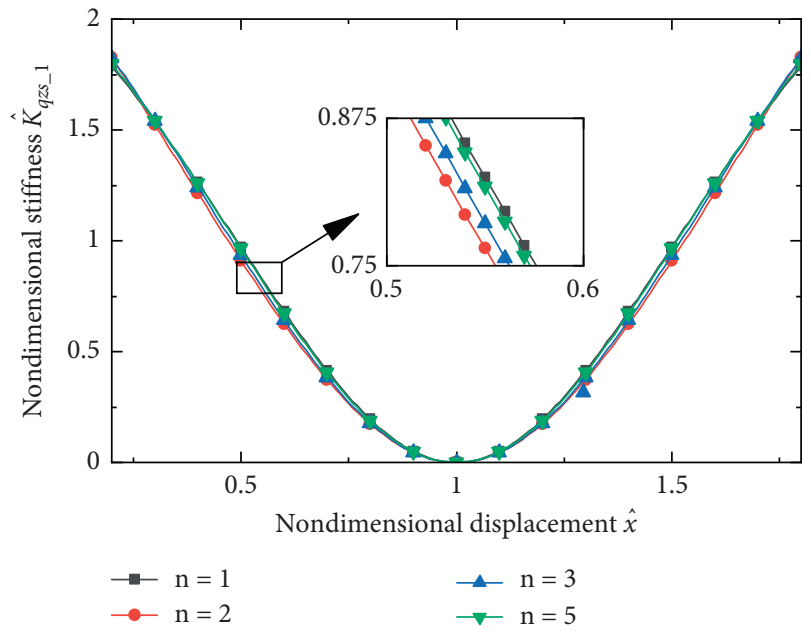

(d)

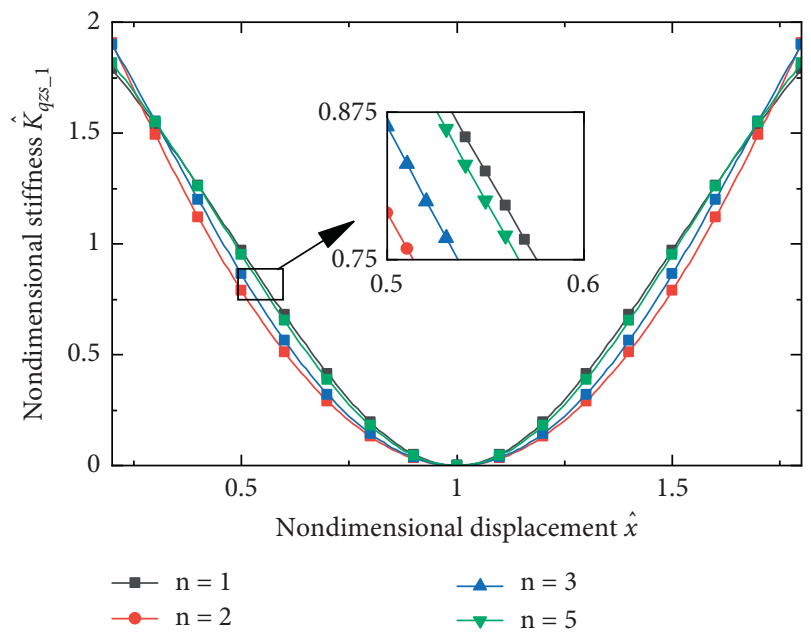

(f)

FIGURE 11: Nondimensional stiffness versus nondimensional displacement for oblique springs with different variable stiffnesses. (a) $n=2$, (b) $n=3$, (c) $n=4$, (d) $\beta=0.5$, (e) $\beta=1$, and (f) $\beta=1.5$. 
isolation performance of the mechanism around the equilibrium position can be improved by oblique springs with softening stiffness, and it is gradually improved with the increase in $\beta$ and the optimum $n$ is 2 .

The extended QZS mechanism with pre-compressed and variable stiffness oblique spring is compared with the typical QZS mechanism, as shown in Figure 12. It reveals that the extended QZS mechanism with both prestressed and softening stiffness oblique spring achieves the minimum dimensionless stiffness at the same displacement and any extended QZS mechanism with one or more proper extended parameters has better stiffness behaviour than the corresponding typical one. When $\hat{x}=0.5$, the nondimensional stiffness $\widehat{K}$ of the QZS isolator is 0.7 . To decrease the value of $\widehat{K}$ by $20 \%$, an extended QZS isolator with parameter $\widehat{\delta}=0.35$ or $\beta=0.5, n=2$ can be feasible. If both parameters are used, $66 \%$ reduction in $\widehat{K}$ can be achieved. In summary, the increase in the parameters $\gamma, \widehat{\delta}$, and $\beta$ can improve the vibration isolation performance around the equilibrium position, and the optimal value of $n$ is 2 . Besides, the effect of $\delta$ on stiffness behaviour can be equivalent to that of $\gamma$, and the effect of $\beta$ and $n$ can be equivalent to each other.

\section{Influence of Friction}

The angle of the oblique spring varies during vibration isolation, and the spring can only withstand axial force, so a rotating pair of the support point $C$ and sliding pair moving along with oblique spring should be designed in the practical QZS mechanism. Thus, friction exists when the support point $C$ moves up and down. As the frictional force is proportional to the pressure of the contact surface and the axial force $F_{a}$ of the spring is clearly much greater than the radial force $F_{r}$ in the system, rotational friction caused by the axial force of the oblique spring is the main friction and discussed here. The force diagrams of the rotational pair and the support point $C$ are analysed, as shown in Figures 13(a) and $13(\mathrm{~b})$.

The normal force $F_{n}$ and the tangential force $F_{t}$ of the contact surface at the hinged end $C$ in Figure 13(a) satisfy the following equation:

$$
F_{t}=\mu F_{n},
$$

where $\mu$ is the coefficient of rotational friction. The support force of the QZS mechanism thus yields the following equation:

$$
F_{d}=F_{0}+2 F_{n} \sin \theta+2 F_{t} \cos \theta
$$

where $F_{\mathrm{t}}$ is a positive value when the support point $C$ moves downward. It can be seen that the term involving friction in equation (32) is a variable about displacement, and the nondimensional stiffness of the system is related to the rotational friction, which can be derived as follows:

$$
\widehat{K}_{d}=1+2 \alpha\left\{1-\frac{\sqrt{1+\gamma^{2}}[1-\mu(\gamma-\widehat{x})]}{\left[1+(\gamma-\hat{x})^{2}\right]^{3 / 2}}\right\} \text {. }
$$

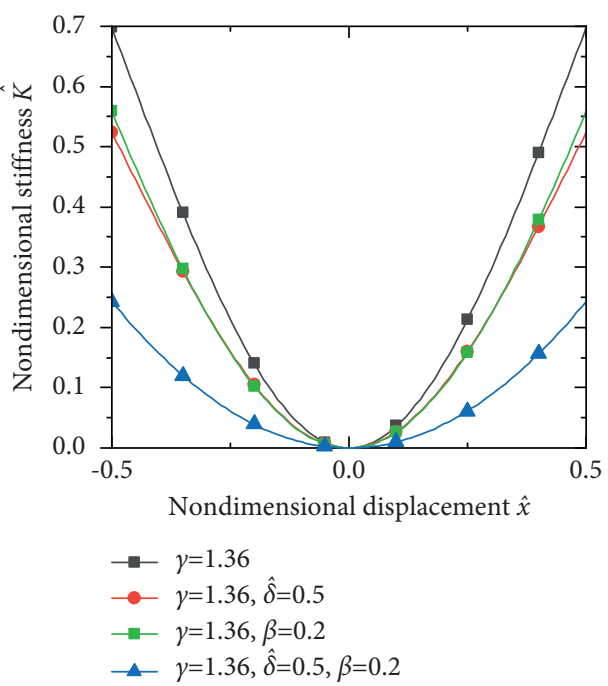

FIgURE 12: Nondimensional stiffness versus nondimensional displacement for the QZS isolators with unstressed (black line), prestressed (red line), variable stiffness (green line), and prestressed and variable stiffness (blue line) oblique springs $(n=2)$.

Equation (33) reveals that the minimum value of $\widehat{K}_{d}$ is not at the equilibrium point. As the extreme value of $\widehat{K}_{d}$ is at the point where the derivative value with respect to $x$ is zero, the corresponding $\hat{x}_{d}$ near the equilibrium position is obtained as follows:

$$
\widehat{x}_{d}=\gamma-\frac{3-\sqrt{8 \mu^{2}+9}}{4 \mu} .
$$

The value of $\widehat{K}_{d}$ should be always a nonnegative value to avoid negative stiffness. In other words, the minimum nondimensional stiffness $\widehat{K}_{d_{-} \text {min }}$ satisfies the following equation:

$$
\widehat{K}_{d_{-} \min }=1+2 \alpha\left(1-\Phi \sqrt{1+\gamma^{2}}\right) \geq 0
$$

where the intermediate parameter $\Phi$ satisfies the following equation:

$$
\Phi=\frac{16 \mu^{3}\left(1+\sqrt{8 \mu^{2}+9}\right)}{\left[6\left(4 \mu^{2}+3-\sqrt{8 \mu^{2}+9}\right)\right]^{3 / 2}} .
$$

When the minimum nondimensional stiffness $\widehat{K}_{d_{-} \min }$ equals zero, the relationship of $\gamma, \alpha$, and $\mu$ in QZS mechanism containing friction is obtained as follows:

$$
\alpha_{\mathrm{qzs} \_d}=\frac{1}{2\left(\Phi \sqrt{1+\gamma^{2}}-1\right)} .
$$

The nondimensional stiffness of the QZS mechanism at the equilibrium position $\widehat{K}_{e_{-} d}$ can be derived as follows: 


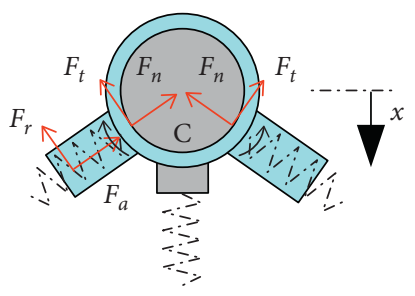

(a)

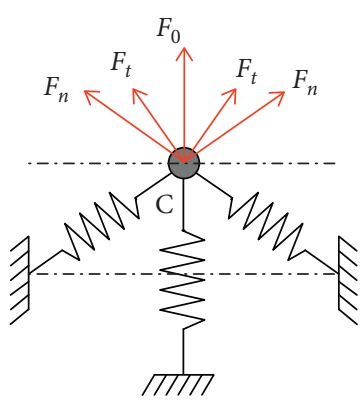

(b)

FIgURE 13: (a) Force diagram of the rotating pairs at the common hinged end of the QZS mechanism. (b) Force diagram of the support point $C$.

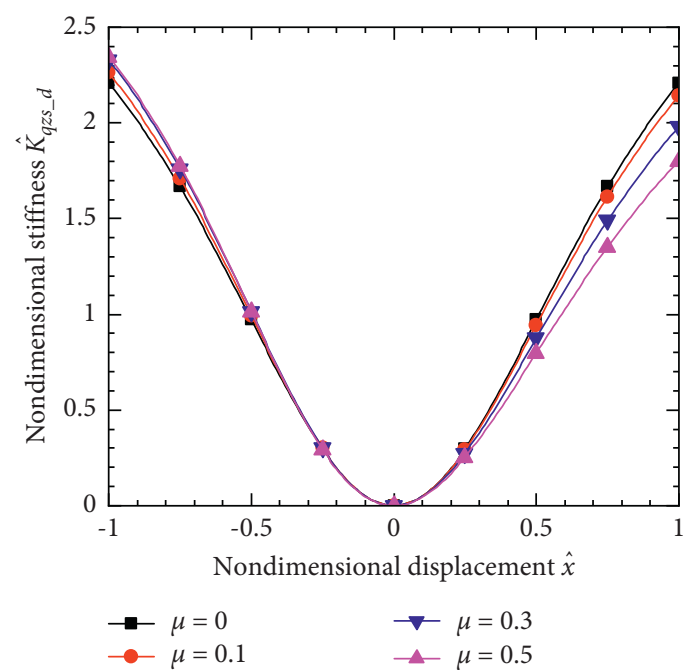

(a)

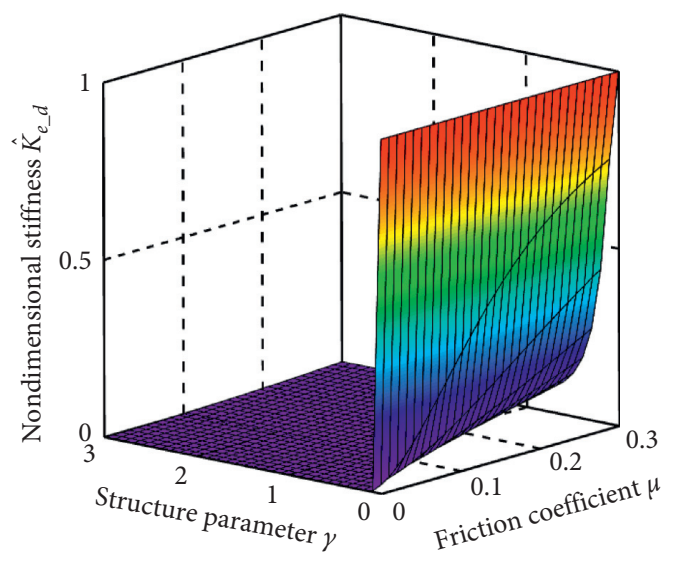

(b)

Figure 14: (a) Nondimensional stiffness versus nondimensional displacement with $\gamma=1$. (b) Nondimensional stiffness of the QZS mechanism at the equilibrium position varies with structure parameter and coefficient of rotational friction.

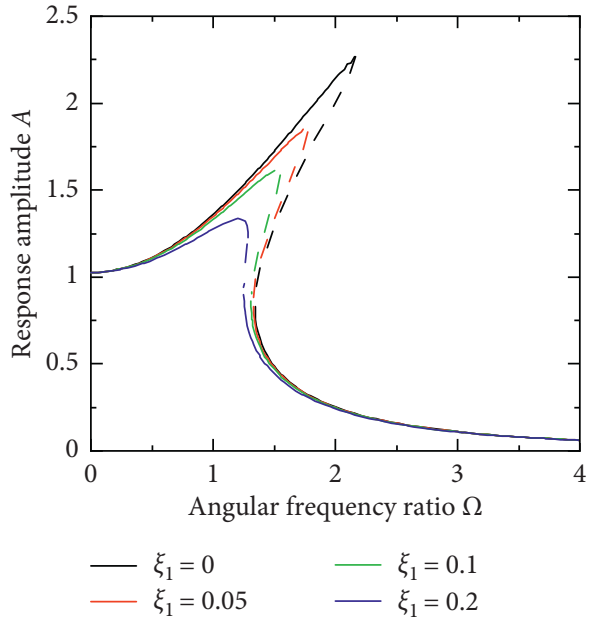

FIGURE 15: Response amplitude versus angular frequency ratio for different friction damping when $\widehat{F}=1$ and $\xi=0.1$.

$$
\widehat{K}_{e_{-} d}=1-\frac{1-\sqrt{1+\gamma^{2}}}{1-\Phi \sqrt{1+\gamma^{2}}} .
$$

The curves of $\widehat{K}_{\mathrm{qzs} \_d}$, denoting the value of $\widehat{K}_{d}$ under the condition of the QZS mechanism, with respect to $\hat{x}$, are plotted in Figure 14(a). For the sake of comparison, the minimum values of $\widehat{K}_{d}$ with different $\mu$ are adjusted to $\widehat{x}=0$. It is shown that the slope of the curves decreases with the increase in $\mu$ when $\widehat{x}$ is greater than $\hat{x}_{d}$, and the result is the opposite when $\hat{x}$ is less than $\hat{x}_{d}$. Besides, Figure 14(b) demonstrates that $\widehat{K}_{e_{-} d}$ is determined by $\gamma$ and $\mu$. $\widehat{K}_{e_{-} d}$ varies greatly with $\mu$ and $\gamma$ when $\gamma$ is less than 1 , and $\widehat{K}_{e_{-} d}$ changes little when $\gamma$ is greater than 1 . As discussed in Section 2.1, the optimal value of $\gamma$ is 1.36 when $L_{0}$ is a constant in the range $(0,1]$. The actual value of $\mu$ is usually not greater than 0.1 , and then, the corresponding $\widehat{K}_{e_{-} d}$ is not greater than 0.004 , which has a very litter impact on the nondimensional stiffness. 
Apart from the effect on stiffness, the friction also generates friction damping, which affects the dynamic characteristic. Assuming the damping ratio caused by friction is $\xi_{1}$, equation (17) becomes as follows:

$$
\widehat{F}^{2}=\left(-\Omega^{2} A+\frac{3}{4} b_{2} A^{3}\right)^{2}+\left[2\left(\xi+\xi_{1}\right) \Omega A\right]^{2} .
$$

The response amplitude curves with different $\xi_{1}$ are drawn in Figure 15. It demonstrates that the damping ratio can affect both peak response amplitude and resonant frequency. As $\xi_{1}$ increases, more energy is dissipated and thus the response amplitude and resonant frequency decrease. The damping ratio is related to the coefficient of friction and force of oblique spring, which can be determined by experiment test.

As a result, the friction changes the zero-stiffness point location that is no longer at the static equilibrium position, but it has little influence on the stiffness characteristic around the static equilibrium position. Besides, friction damping caused by friction affects the dynamic characteristic. The response amplitude and resonant frequency decrease as the parameter $\xi_{1}$ increases. Furthermore, considering the errors of structural parameters and avoiding the existence of a negative stiffness region in the practical mechanism, a similar analysis can be conducted and the isolation performance can be investigated.

\section{Conclusion}

An improved optimization method for the structural parameters of a typical QZS isolator with three springs has been employed in this study. The optimization criteria of the QZS mechanism have been proposed to obtain the corresponding optimum parameters by achieving the maximum nondimensional displacement from the equilibrium position without exceeding a given natural frequency and having as great a load range as possible. Either of the vibration isolation characteristics and load capacity can be improved by adjusting the geometric parameter at the expense of the other one. When the length of the oblique spring is a constant and less than $1 \mathrm{~m}$, the optimum geometric parameter is 1.36 , and the results show better vibration isolation performance around the equilibrium position in the range of both linear behaviour and nonlinear behaviour.

Further qualitative analysis of an extended QZS isolator consisting of vertical fixed stiffness and prestressed oblique springs with variable stiffness has been conducted, and it reveals that prestressed oblique springs with softening stiffness can improve the stiffness property and the best exponent of the nonlinear stiffness is 2 . Besides, the isolator with multiple extended parameters has better stiffness characteristic than one with single extended parameters.

Finally, the influence of friction is analysed in the practical QZS mechanism. The friction has little influence on the stiffness characteristic of the QZS mechanism around the static equilibrium position. Meanwhile, the damping ratio caused by friction can affect both peak response amplitude and resonant frequency.

\section{Data Availability}

The data used to support the findings of this study are included within the article.

\section{Conflicts of Interest}

The authors declare that there are no conflicts of interest regarding the publication of this study.

\section{Acknowledgments}

This work was supported by the Wuhan Science and Technology Plan (no. 2019010702011269).

\section{References}

[1] P. M. Alabuzhev and E. I. Rivin, Vibration Protecting and Measuring Systems with Quasi-Zero Stiffness, Hemisphere Pub. Corp., New York, 1989.

[2] A. Carrella, M. J. Brennan, and T. P. Waters, "Static analysis of a passive vibration isolator with quasi-zero-stiffness characteristic," Journal of Sound and Vibration, vol. 301, no. 3-5, pp. 678-689, 2007.

[3] I. Kovacic, M. J. Brennan, and T. P. Waters, "A study of a nonlinear vibration isolator with a quasi-zero stiffness characteristic," Journal of Sound and Vibration, vol. 315, no. 3, pp. 700-711, 2008.

[4] A. Carrella, M. J. Brennan, I. Kovacic, and T. P. Waters, "On the force transmissibility of a vibration isolator with quasizero-stiffness," Journal of Sound and Vibration, vol. 322, no. 45, pp. 707-717, 2009.

[5] X. Wang, H. Liu, Y. Chen, and P. Gao, "Beneficial stiffness design of a high-static-low-dynamic-stiffness vibration isolator based on static and dynamic analysis," International Journal of Mechanical Sciences, vol. 142-143, pp. 235-244, 2018.

[6] X. Liu, X. Huang, and H. Hua, "Performance of a zero stiffness isolator under shock excitations," Journal of Vibration and Control, vol. 20, no. 14, pp. 2090-2099, 2014.

[7] Y. Wang, S. Li, and C. Cheng, "Investigation on a quasi-zerostiffness vibration isolator under random excitation," Journal of Theoretical and Applied Mechanics, vol. 54, no. 2, pp. 621-632, 2016.

[8] G. Gatti, "A K-shaped spring configuration to boost elastic potential energy," Smart Materials and Structures, vol. 28, no. 7, Article ID 77002, 2019.

[9] F. Zhao, J. C. Ji, K. Ye, and Q. Luo, "Increase of quasi-zero stiffness region using two pairs of oblique springs," $\mathrm{Me}$ chanical Systems and Signal Processing, vol. 144, Article ID 106975, 2020.

[10] F. Zhao, J. Ji, Q. Luo, S. Cao, L. Chen, and W. Du, “An improved quasi-zero stiffness isolator with two pairs of oblique springs to increase isolation frequency band," Nonlinear Dynamics, vol. 104, no. 1, pp. 349-365, 2021.

[11] F. Zhao, J. Ji, K. Ye, and Q. Luo, "An innovative quasi-zero stiffness isolator with three pairs of oblique springs," International Journal of Mechanical Sciences, vol. 192, Article ID 106093, 2021.

[12] G. Yan, H.-X. Zou, S. Wang et al., "Large stroke quasi-zero stiffness vibration isolator using three-link mechanism," Journal of Sound and Vibration, vol. 478, Article ID 115344, 2020. 
[13] D. L. Thanh and A. D. N. Vu, "Low frequency vibration isolator with adjustable configurative parameter," International Journal of Mechanical Sciences, vol. 134, pp. 224-233, 2017.

[14] Z. Lu, M. J. Brennan, T. Yang, X. Li, and Z. Liu, “An investigation of a two-stage nonlinear vibration isolation system," Journal of Sound and Vibration, vol. 332, no. 6, pp. 1456-1464, 2013.

[15] Z. Lu, T. Yang, M. J. Brennan, X. Li, and Z. Liu, "On the performance of a Two-Stage vibration isolation system which has geometrically nonlinear stiffness," Journal of Vibration and Acoustics-Transactions of the ASME, vol. 136, no. 6, Article ID 064501, 2014.

[16] Z. Lu, T. Yang, M. J. Brennan, Z. Liu, and L. Chen, "Experimental investigation of a Two-Stage nonlinear vibration isolation system with High-Static-Low-Dynamic stiffness," Journal of Applied Mechanics-Transactions of the Asme, vol. 84, no. 0210012, 2017.

[17] Y. Wang, S. Li, S. A. Neild, and J. Z. Jiang, "Comparison of the dynamic performance of nonlinear one and two degree-offreedom vibration isolators with quasi-zero stiffness," Nonlinear Dynamics, vol. 88, no. 1, pp. 635-654, 2017.

[18] A. Abolfathi, M. J. Brennan, T. P. Waters, and B. Tang, "On the effects of mistuning a Force-Excited system containing a Quasi-Zero-Stiffness vibration isolator," Journal of Vibration and Acoustics-Transactions of the ASME, vol. 137, no. 0445024, p. 44502, 2015.

[19] A. D. Shaw, S. A. Neild, and M. I. Friswell, "Relieving the effect of static load errors in nonlinear vibration isolation mounts through stiffness asymmetries," Journal of Sound and Vibration, vol. 339, pp. 84-98, 2015.

[20] L. Meng, J. Sun, and W. Wu, "Theoretical design and characteristics analysis of a Quasi-Zero stiffness isolator using a disk spring as negative stiffness element," Shock and Vibration, vol. 2015, no. 4, pp. 1-19, 2015.

[21] Y. Zhou, P. Chen, and G. Mosqueda, "Analytical and numerical investigation of Quasi-Zero stiffness vertical isolation system," Journal of Engineering Mechanics, vol. 145, no. 6, 2019.

[22] K. Kim, Y. You, and H. Ahn, "Optimal design of a QZS isolator using flexures for a wide range of payload," International Journal of Precision Engineering and Manufacturing, vol. 14, no. 6SI, pp. 911-917, 2013.

[23] G. Dong, X. Zhang, Y. Luo, Y. Zhang, and S. Xie, "Analytical study of the low frequency multi-direction isolator with high-static-low-dynamic stiffness struts and spatial pendulum," Mechanical Systems and Signal Processing, vol. 110, pp. 521-539, 2018.

[24] Y. Zheng, Q. Li, B. Yan, Y. Luo, and X. Zhang, "A Stewart isolator with high-static-low-dynamic stiffness struts based on negative stiffness magnetic springs," Journal of Sound and Vibration, vol. 422, pp. 390-408, 2018.

[25] Y. Sun, J. Zhao, M. Wang et al., "High-static-low-dynamic stiffness isolator with tunable electromagnetic mechanism," IEEE, vol. 25, no. 1, pp. 316-326, 2020.

[26] X. Liu, X. Huang, and H. Hua, "On the characteristics of a quasi-zero stiffness isolator using Euler buckled beam as negative stiffness corrector," Journal of Sound and Vibration, vol. 332, no. 14, pp. 3359-3376, 2013.

[27] B. A. Fulcher, D. W. Shahan, M. R. Haberman, C. C. Seepersad, and P. S. Wilson, "Analytical and experimental investigation of buckled beams as negative stiffness elements for passive vibration and shock isolation systems,"
Journal of Vibration and Acoustics-Transactions of the ASME, vol. 136, no. 0310093, p. 31009, 2014.

[28] H. Ding and L.-Q. Chen, "Nonlinear vibration of a slightly curved beam with quasi-zero-stiffness isolators," Nonlinear Dynamics, vol. 95, no. 3, pp. 2367-2382, 2019.

[29] J. Zhou, X. Wang, D. Xu, and S. Bishop, "Nonlinear dynamic characteristics of a quasi-zero stiffness vibration isolator with cam-roller-spring mechanisms," Journal of Sound and Vibration, vol. 346, no. 1, pp. 53-69, 2015.

[30] H.-J. Ahn, S.-H. Lim, and C. Park, "An integrated design of quasi-zero stiffness mechanism," Journal of Mechanical Science and Technology, vol. 30, no. 3, pp. 1071-1075, 2016.

[31] M. Li, W. Cheng, and R. Xie, "Design and experiments of a quasi-zero-stiffness isolator with a noncircular cam-based negative-stiffness mechanism," Journal of Vibration and Control, vol. 26, no. 107754632090868921-22, pp. 1935-1947, 2020.

[32] X. Sun and X. Jing, "Multi-direction vibration isolation with quasi-zero stiffness by employing geometrical nonlinearity," Mechanical Systems and Signal Processing, vol. 62-63, pp. 149-163, 2015.

[33] W. Zhang and J. Zhao, "Analysis on nonlinear stiffness and vibration isolation performance of scissor-like structure with full types," Nonlinear Dynamics, vol. 86, no. 1, pp. 17-36, 2016.

[34] W. Zou, C. Cheng, R. Ma, Y. Hu, and W. Wang, "Performance analysis of a quasi-zero stiffness vibration isolation system with scissor-like structures," Archive of Applied Mechanics, vol. 91, no. 1, pp. 117-133, 2020.

[35] Z. Zhou, Y. Gao, L. Sun, W. Dong, and Z. Du, “A bistable mechanism with linear negative stiffness and large in-plane lateral stiffness: design, modeling and case studies," Mechanical Sciences, vol. 11, no. 1, pp. 75-89, 2020.

[36] Z.-Q. Lu, D.-H. Gu, H. Ding, W. Lacarbonara, and L.-Q. Chen, "Nonlinear vibration isolation via a circular ring," Mechanical Systems and Signal Processing, vol. 136, Article ID 106490, 2020.

[37] D. Zou, G. Liu, Z. Rao, T. Tan, W. Zhang, and W.-H. Liao, “A device capable of customizing nonlinear forces for vibration energy harvesting, vibration isolation, and nonlinear energy sink," Mechanical Systems and Signal Processing, vol. 147, Article ID 107101, 2021.

[38] Z. Hao, Q. Cao, and M. Wiercigroch, "Two-sided damping constraint control strategy for high-performance vibration isolation and end-stop impact protection," Nonlinear Dynamics, vol. 86, no. 4SI, pp. 2129-2144, 2016.

[39] X. Sun, X. Jing, J. Xu, and L. Cheng, "Vibration isolation via a scissor-like structured platform," Journal of Sound and Vibration, vol. 333, no. 9, pp. 2404-2420, 2014.

[40] X. Sun and X. Jing, "Analysis and design of a nonlinear stiffness and damping system with a scissor-like structure," Mechanical Systems and Signal Processing, vol. 66-67, pp. 723-742, 2016.

[41] H.-J. Ahn, "A variable stiffness mechanism for a movable magnet track of a linear motor stage," Journal of Mechanical Science and Technology, vol. 31, no. 11, pp. 5203-5207, 2017.

[42] M. Li, W. Cheng, and R. Xie, "Design and experimental validation of a cam-based constant-force compression mechanism with friction considered," Proceedings of the Institution of Mechanical Engineers - Part C: Journal of Mechanical Engineering Science, vol. 233, no. 11, pp. 3873-3887, 2018. 